\title{
El rastreo con mamografía entre los 70 y 79 años no resultó costo-efectivo
}

Continuing screening mammography in women aged 70 to 79 years: impact on life expectancy and cost-effectiveness. Kerlikowske K; Salzman P; Philips K et al. JAMA 1999; 282: $2156-2163$.

Objetivo

Comparar la expectativa de vida y la relación costo-efectividad de tres estrategias de rastreo mamográfico en mujeres entre 70 y 79 años.

\section{Diseño}

Estudio de análisis de decisión y costo-efectividad, utilizando el modelo de Markov*.

\section{Muestra}

Población general de 10.000 mujeres de 65 años de edad.

\section{Intervención}

El análisis evaluó la comparación de 3 estrategias: a) Mamografía bienal desde los 65 años a los 69 años de edad y luego discontinuar el rastreo. b) Mamografía bienal de los 65 a los 69 años de edad y medición de la densidad mineral sea (DM0) en la porción distal del radio a los 65 años. Sólo en aquellas mujeres con DM0 en los tres cuartilos superiores el modelo asume continuar el rastreo hasta los 79 años. c) Mamografía bienal desde los 65 a los 79 años.

Aclaración: Se comparan estas estrategias debido a que la exposición acumulativa a estrógenos endógenos se ha asociado a un riesgo elevado de carcinoma de mama, y que la DMO es un marcador de dicha exposición. Ha sido demostrado que el hallazgo de una densidad elevada es un fuerte predictor del riesgo de cáncer de mama.

\section{Medida de Resultados y Costos Principales}

Se utilizó el número de muertes evitadas por cáncer de mama, la expectativa de vida y el incremento en la relación costo efectividad. ALgunos costos incluidos: mamografía $\$ 116$; costo promedio de un falso positivo: \$379; tratamiento del carcinoma invasor con y sin ras- treo: $\$ 36.866$ y 38.713 respectivamente; tratamiento del carcinoma ductal in situ en ambos grupos: $\$ 26.667$.

\section{Resultados}

Teniendo en cuenta el escenario de incluir la DMO como modificador de la estrategia de rastreo, los autores compararon la primer estrategia (rastreo solamente de los 65 a 69 años) frente a medir la DM0 a los 65 años en 10.000 mujeres y continuar el rastreo más allá de los 69 sólo en aquellas de los tres cuartilos superiores de DMO. Esta estrategia prevendría 9,4 muertes y adicionaría en promedio 2,1 días a la expectativa de vida. El incremento en la relación costo efectividad sería de $\$ 66.773$ por año de vida salvado. Para prevenir una muerte por cáncer de mama, 1064 mujeres deberían participar del rastreo, lo que implica aproximadamente 2.011 mamografias adicionales por muerte evitada.

Continuar con el rastreo bienal mamográfico (la tercer estrategia) en las 10.000 mujeres hasta los 79 años, evitaría 1,4 muertes más que el segundo esquema y agregaría 7,2 horas a la expectativa de vida. El incremento en el cociente costo efectividad de la intervención seria de $\$ 117.689$ por año de vida salvado. Para prevenir una muerte adicional, 7.143 mujeres deberían reclutarse bajo este esquema y en comparación con la anterior estrategia, resultaría en 9.963 mamografías adicionales.

\section{Conclusiones}

El análisis sugiere que los beneficios en la expectativa de vida y la relación costo efectividad de continuar el rastreo luego de los 69 años en mujeres con elevada DMO es mínimo. El impacto de rastrear a todas las mujeres en este grupo etario sería costoso y no significativo

\section{COMENTARIO}

Aunque no existe un umbral estricto más allá del cualuna estrategia se considera costo-efectiva, en salud pública sueteri considerarse costoefectivas aquellas intervenciones cuyo costo es menor a $\$ 50.000$ por año de vida salvado. Entre $\$ 50.000$ y $\$ 100.000$ se encuentra en el terreno de los grises, y más allá de los $\$ 100.000$ no se considera costo-efectiva. ${ }^{1}$ Las estrategias que intenta evaluar el presente estudio tienen un bajo impacto en la expectativa de vida y un alto costo extra; esto se debe a que el cáncer de mama no representa una de las causas principales de muerte en este grupo etario. Además la baja expectativa de vida en las mujeres de más de 70 años, disminuye la relación costo efectividad de las intervenciones. Por otro lado, el rastreo tiende a diagnosticar carcinomas tempranos que nunca producirán síntomas ni afectará la expectativa de vida, pero incrementará la frecuencia de tratamientos quirúrgicos. ${ }^{2}$ El $8 \%$ de estas mujeres tendrá una mamografía patológica, pero sólo el 7 al 15\% de ellas tendrá un carcinoma. ${ }^{3}$
La mayor limitante del estudio es que la eficacia del rastreo mamográfico es controvertido. Aún es débil, la evidencia sobre el efecto del rastreo en la reducción global de la tasa de mortalidad por cáncer de mama obtenido de estudios randomizados. Quizás la mejor información disponible hasta el momento es que el rastreo prevendría 1 muerte por cáncer de mama de cada 1.000 mujeres reclutadas. ${ }^{4}$ En la República Argentina mueren por año 4989 mujeres por esta causa. El $40 \%$ de ellas tiene entre 50 y 69 años, el $24 \%$ entre 70 y 79 y el $21 \%$ mas de 79 . Sin embargo, las muertes por cáncer de mama solo representan el $4 \%$ de las muertes totales en el grupo entre 50 y 69 , y el $3 \%$ en el grupo de 70 op más. ${ }^{5}$

Por ello, las políticas de salud orientadas a la disminución de la mortalidad por carcinoma de mama en nuestro país, no solo debieran considerar la relación costo efectividad de la intervención en los distintos grupos etarios, sino la prevalencia de la enfermedad y el acceso a la salud.

*Ver glosario

Dr. Hugo B. Krupitzki

Departamento de Ginecología y Obtetricia. CEMIC

\footnotetext{
Referencias

1. Laupacis A, Feeny D, Detsky AS, et al. How attractive does a new technology have to be to warrant adoption and utilization ?. CMAJ. 1992; 146: 473-481

2. Welch HG, Fisher ES, Diagnostic testing following screening mammography in the elderly. J. Natl Cancer Inst. 1998; 90:1389-1392

3. Brown M. Economic consideration in breast cancer screening of older women. J Gerontol. 1992; 47: 51-58

4. Gatzsche PC, Olsen, 0. Is screening for breast cancer with mammography justifiable? Lancet 2000; 355: 129-34

5. Estadisticas Vitales 1998. Publicación oficial. Ministerio de Salud de la República Argentina. Diciembre de 1999
} 